Research Article

iMedPub Journals

http://www.imedpub.com

Vol. 1 No. 1:2

DOI: $10.21767 / 2471-8157.10002$

\section{Evaluating the Association between Anthropometric Indices and Cardiovascular Risk Factors with Symptomatic Peripheral Arterial Disease in Type 2 Diabetes Mellitus}

\section{Abstract}

Objectives: The main aim of the study was to find the association between obesity and other anthropometric indices with symptomatic peripheral arterial disease (PAD) in type 2 diabetes mellitus (T2DM) patients. Furthermore, we investigated other risk factors of symptomatic PAD in T2DM.

Study Design: In a case-control design, 46 cases and 69 controls were enrolled, aged between 40 and 75 years. Cases were age and gender matched with controls. Ankle-brachial index $(A B I)<0.9$ was a proxy for PAD in T2DM patients. Anthropometric indices were measured and known cardiovascular risk factors were collected, area under curve (AUC) and logistic regression were applied.

Results: Hypertensive patients had 2.5 fold higher risk of symptomatic PAD than normotensive patients, (CI 95\%=1.16-5.51). Smoking habit was remarkably higher in cases than controls, $\mathrm{OR}=8.2 \mathrm{Cl} 95 \%$ (3.2-8.2), however this was not significant in smoking subgroups based on duration of smoking (pack-year). Waste hip ratio (WHR) $\geq 1$ in both male and female was significantly related to the outcome, Odds ratio 3.12 ( $\mathrm{Cl} 95 \% 1.25-7.82)$ in men and of 26.67 ( $\mathrm{Cl} 95 \% 3.77-188.51)$ in women. In the final model (forward stepwise regression), WHR $\geq 1$, hypertension, duration of DM and Smoking were significantly related to symptomatic PAD.

Conclusion: As obese patients are more prone to type 2 diabetes mellitus, the previous cut-offs of obesity may change in the future to assess the risk of CVD in this group. We suggest that WHR is a simple measure of abdominal obesity and is related to symptomatic PAD.

Keywords: Obesity, Peripheral arterial disease

Received: September 15, 2015; Accepted: October 19, 2015; Published: October 28,2015

\section{Introduction}

Peripheral artery disease (PAD) in terms of atherosclerosis of lower extremity vessels below aortic bifurcation is considered to be an important marker of cardiovascular disease. Increasing prevalence, concomitant morbidity/mortality and misleading symptoms of PAD has urged the physician to increase their awareness about diagnosis and also treatment of PAD. In previous

\author{
Ali Vasheghani-Farahani", \\ Haleh Ashraf², \\ Maryam Abolhasani ${ }^{3}$ \\ Kaveh Hosseini" , Seyyed \\ Aboozar jazayeri 5 and \\ Shahrokh Karbalayi² Center, Tehran University of Medical Sciences, Iran Tehran University of Medical Sciences, Iran Hospital, Tehran University of Medical Sciences, Iran health, Tehran University of Medical Sciences, Iran Tehran University of Medical Sciences, Iran \\ 1 Department of Cardiology, Tehran Heart \\ 2 Department of Cardiology, Sina Hospital, \\ 3 Department of Sport Medicine, Sina \\ 4 General Practitioner, Master of public \\ 5 Laboratory Manager, Sina Hospital,
}

Corresponding author: Kaveh Hosseini

ĐKaveh_hosseini130@yahoo.com

General Practitioner, Master of Public Health, Tehran Heart Center, Tehran University of Medical Sciences, Jalal Al Ahmad and North Kargar Cross, Tehran, Iran.

Tel: +989113538440

Citation: Vasheghani-Farahani A, Ashraf $\mathrm{H}$, Abolhasani $\mathrm{M}$, et al. Evaluating the Association between Anthropometric Indices and Cardiovascular Risk Factors with Symptomatic Peripheral Arterial Disease in Type 2 Diabetes Mellitus. Interv Cardiol J 2015, 1:1. 
diabetes mellitus (T2DM) patients with T2DM foot [4] are reported for PAD detection by this method.

Escalating number of T2DM patients around the world has made T2DM a major public health issue in Iran [5]. Two to fourfold increased risk of PAD incident in T2DM patients compared with general population [6], and poor outcomes of this disease in T2DM patients, obligate physicians and researchers to take immediate measures. The great number of PAD patients remains undiagnosed due to asymptomatic course of the disease or atypical symptoms [6]. Claudication, at rest calf pain and diminished or absent lower extremity pulses are among the most prevalent symptoms and signs of PAD. The severity and location of the symptoms and signs are related to the degree of stenosis and the site of the culprit lesion, respectively.

Many of traditional risk factors of cardio-vascular disease play an important role here, such as Hypertension (HTN), Dyslipidemia (DLP) and Smoking [7]. Inflammation/coagulation biomarkers like high sensitivity C-reactive protein (hs-CRP), fibrinogen and D-dimer may also be associated with PAD and/or may worsen the outcome of the disease [8].

Excessive numbers of overweight and obese people around the world and proven association of excess adiposity with CVD risk factors remind us to be always aware of different management and preventive strategies of obesity [8]. In T2DM patients, the overall prevalence of overweight and obesity is approximately $80 \%$ [9]. Body mass index (BMI) as an easy way to measure general obesity has been challenged by recent researches. Its limitation to differentiate lean and fat mass and also lack of accuracy to represent visceral fat distribution made this index less important than others [8]. Abdominal obesity is far more important than general obesity in causing atherosclerosis of great vessels and resulting $P A D$, myocardial infarction and cerebrovascular accident [10]. In previous study by VaSheghani et al., new index was defined to be remarkably related to cardiovascular disease, as the proportion of sagittal anterio posterior diameter to triceps skinfold [11]. Waist circumference (WC), waist-hip-ratio (WHR) and waist-height ratio (WHtR) are other reported indices which indicate abdominal obesity $[12,13]$. In recent studies, WC and WHtR were associated with T2DM $[12,14]$. Debates are still continuing about the effects of anthropometric variables on CVD risk factors, especially T2DM [15].

\section{Materials and Methods}

The study included 46 cases and 69 controls, aged between 40 and 75 years old, and was conducted between March 2014 and August 2015. Cases and controls were matched according to gender and Age. We did not match the cases and controls based on their weight and height in order to preclude the overmatching bias. Cases were T2DM candidates of peripheral angiography referred to endovascular surgery clinic, and controls were among T2DM patients in urology department and also T2DM patients coming to diabetes day clinic for routine check-up.

Diagnosis of PAD was based on $A B I \leq 0.9$, using $8 \mathrm{MHz}$ duplex sonogram probe (Bestos co. Ltd. Made in Korea). This Index was the ratio of the highest systolic pressure of the ankles to the highest systolic pressure of the arms [2]. In this case-control design, cases with T2DM and symptomatic PAD were enrolled and controls were among T2DM patients without PAD (ABI>0.9). Patients with $A B \mid>1.3$ or non-compressible arteries were excluded. In addition cases with the history of angioplasty in the same leg which was symptomatic were not included. Diabetic patients with symptomatic $P A D$ which had $A B I \leq 1.3$ and were newly diagnosed as PAD patients were included.

Basic and demographic information (age, gender, smoking habit, T2DM duration, hypertension and dyslipidemia) were collected using a questionnaire designed by the researchers.

Anthropometric indices (BMI, WC, HC, WHR, WHtR, Thigh Circumference (TC) and waist thigh ratio (WTR)) were measured by a single trained examiner. Weight with a light clothing and height without shoes were measured. BMI was then calculated as weight divided by height squared $\left(\mathrm{kg} / \mathrm{m}^{2}\right)$. Non-stretchable tape around the waist at the level of the iliac crest at the end of normal expiration (WC), around the hip at the greatest diameter of the buttock $(\mathrm{HC})$ and around the thigh at the midpoint level perpendicular to the long axis of the thigh (TC) were all measured by same investigator. Anthropometrics were categorized as follows: In men, overweight (BMI of $25-29.9 \mathrm{~kg} / \mathrm{m}^{2}$, WC of 94 $101.9 \mathrm{~cm}$ and WHR of 0.9-0.99), obese (BMI $\geq 30 \mathrm{~kg} / \mathrm{m}^{2}, W C \geq$ 102 and $W H R \geq 1$ ) in women overweight (BMI of $25-29.9 \mathrm{~kg} / \mathrm{m}^{2}$, WC of $80-87.9 \mathrm{~cm}$ and WHR of $0.80-0.84$ ), obese (BMI $\geq 30 \mathrm{~kg} /$ $\mathrm{m}^{2}, \mathrm{WC} \geq 0.088$ and $\left.W H R \geq 0.88\right)[14,16]$ and $W H t R \geq 0.5$ [17]. The method of receiver operating characteristic (ROC) curves was used in by plotting true positive (sensitivity) against false positive (1-specificity) rates, to determine the cut-off values for WHR. The cut off value was the point with maximal sensitivity and specificity [18].

Patients were asked to fast for at least 8 hours. Laboratory tests included lipid profile (Total Cholesterol (TC), High density lipoprotein (HDL), Low density lipoprotein (LDL) and Triglycerides (TG)), Fasting Blood Sugar (FBS), HbA1C and hs-CRP.

Duration of T2DM was another variable which was classified in three groups, $<5$ years, 5 to 10 years and $>10$ years. Hypertensive patients were defined as systolic blood pressure (BP) $\geq 140$ $\mathrm{mmHg}$ and/or diastolic BP $\geq 90 \mathrm{mmHg}$ and/or normal BP under medication). The cut-off points for dyslipidemia were: $T C \geq 240$ $\mathrm{mg} / \mathrm{dl}, \mathrm{TG} \geq 240 \mathrm{mg} / \mathrm{dl}, \mathrm{HDL}<40$ and LDL $\geq 160$ [19]. Normal amounts of each under medication were also considered as dyslipidemia. Diabetes diagnosis was confirmed by both $\mathrm{HbA} 1 \mathrm{C}$ $\geq 7$ and/or two fasting blood sugar $\geq 126$. Chemiluminescence method (SNIBE company, Maglumi 2000) was used to detect hsCRP quantitatively, with normal range up to $700 \mathrm{ngr} / \mathrm{ml}$. Tobacco smoking was also reported in 4 levels, none, $<10$ pack-year ( $p / y r)$, $10-20 \mathrm{p} / \mathrm{yr}$ and $>20 \mathrm{p} / \mathrm{yr}$.

\section{Statistical analysis}

All data were analyzed by SPSS software version 14 (IBM SPSS Chicago). Categorical variables were reported as frequencies and compared using Pearson chi-square. Continuous variables were evaluated with Independent sample T-test. One-way Anova was used to compare means between groups. P-value less than 0.05 considered to be statistically significant. Logistic regression was 
applied to calculate crude and adjusted effect of every variable on the outcome of the interest. Enter and Step forward stepwise methods in regression were used to extract the best fitted equation based on known CVD risk factors. Receiver Operating Characteristic (ROC) analyses were used to determine the area under ROC curves (AUC) between each anthropometric index and PAD. The cut off value was the point with maximal sensitivity and specificity.

\section{Results}

Basic demographic features of the patients (cases vs. controls) are shown in Table 1. As shown in Table 1, there was no significant age and gender difference between cases and controls. Significant associations between symptomatic PAD incident and main CVD risk factors in terms of Odds ratio in univariate binary logistic regression are also shown in Table 1. Age, HTN, duration DM and smoking were significant, while dyslipidemia was not ( $p$-value 0.38). Hypertensive patients had 2.5 fold higher risk of symptomatic PAD than normotensive patients, $(\mathrm{Cl}$ $95 \%=1.16-5.51)$. Smoking habit was remarkably higher in cases than controls $(\mathrm{OR}=8.2 \mathrm{Cl} 95 \%(3.2-8.2)$, however this was not significant in smoking subgroups based on duration of smoking. Duration of DM was significantly different between PAD groups ( $p$-value $=0.038)$. The group with $<5$ years DM duration was considered the reference group, and all the ratios were reported based on these consumption. Traditional risk factor of CVD, dyslipidemia, was not an important variable in PAD incident among patients with diabetes. High Sensitive CRP was above the normal range $(<700 \mathrm{ng} / \mathrm{ml})$ in all of them. There were not any significant quantitative difference in hs-CRP between cases and controls ( $p$-value $=0.53$ ).

Anthropometric indices of both groups are depicted and compared, Table 2. Comparing means between cases and controls with Independent Sample T-test and also area under curve (AUC) between each anthropometrics and PAD are shown, Table $\mathbf{2}$. In our study population, weight, BMI and WC were higher in control groups, but statistically insignificant ( $p$-value $>0.05$ ). However the significant difference between two groups was WHR, which was higher in cases. In addition, the only anthropometric index in which the AUC was greater than 0.5 ( $p$-value=0.01), was WHR. Hip Circumference was significantly higher in controls (Mean difference $=10.35$, $p$-value $=0.001$ ). As mentioned above in methods, BMI was categorized in 3 levels (normal, overweight and obese). Distribution of BMI between cases and controls were: in overweight patients (BMI $25-29.9 \mathrm{~kg} / \mathrm{m}^{2}$ ) 16 cases vs 25 controls and in obese patients (BMI $\left.\geq 30 \mathrm{~kg} / \mathrm{m}^{2}\right) 9$ cases vs. 18 controls. None of the BMI subgroups were statistically relevant to the outcome of the interest ( $p$ value 0.055 and 0.061 in obese and overweight group, respectively). Mean age difference between $3 \mathrm{BMl}$ subgroups (BMI $\geq 30,25 \leq \mathrm{BMI}<30$ and $\mathrm{BMI}<25$ ) was not significant, in One-way Anova method ( $p$-value=0.094). Patients with $\mathrm{BMI} \geq 30$ had the mean age of $56.07( \pm 12.67)$, and those with $25 \leq \mathrm{BMI}<30$ had the mean age of $61.86( \pm 11.74)$, $\mathrm{p}$-value $=0.067$.

The ROC was also applied to determine the cut-off values which can better demonstrate the balanced sensitivity and specificity for waist-hip ratio, in male and female, Table 3 . The best cut off value of WHR (maximal sensitivity and specificity) in our study was 1 . In men with T2DM, WHR $\geq 1$ had the sensitivity and specificity of $69 \%$ and $60 \%$ respectively, for PAD detection. Ten women in case group $(n=12)$ and three women in control group $(n=19)$ had WHR $\geq 1$ (Pearson chi-square $17, p$-value $=0.000$ ). Hence, WHR $\geq 1$ in both male and female was significantly related to the outcome, Odds ratio 3.12 (Cl 95\% 1.25-7.82) in men and of 26.67 (Cl 95\% 3.77-188.51) in women. Discriminative ability (based on AUC values) of WHR was significantly higher than BMI in both sexes 0.91 vs. 0.32 in female and 0.60 vs. 0.37 in male patients.

Suggested cut-off for WC in obese patients and metabolic syndrome ( $W C>102 \mathrm{~cm}$ in male and $>88 \mathrm{~cm}$ in female) was also tested in this study that was not significantly related to PAD ( $p$-value $=0.71$ in male and 0.45 in female patients). We also

Table 1 Basic features of both groups, comparison in terms of Pearson Chi-square and binary uni-variate logistic regression.

\begin{tabular}{|c|c|c|c|c|}
\hline & Cases $n=46(\%)$ & Controls $n=69(\%)$ & p-value & Odds Ratio ${ }^{\circledR}$ \\
\hline Female & $12(26 \%)$ & $19(28 \%)$ & 0.66 & \\
\hline Age & $64 \pm 10.0$ & $58 \pm 11.7$ & 0.049 & 1.06 \\
\hline Duration T2DM & & & 0.038 & \\
\hline$<5$ & $8(17.3 \%)$ & $25(36.2 \%)$ & 0.06 & 1 \\
\hline $5-10$ & $14(30 \%)$ & $14(20.2 \%)$ & 0.033 & 4.3 \\
\hline$>10$ & $24(52 \%)$ & $30(50 \%)$ & 0.042 & 3.5 \\
\hline HTN & $31(67 \%)$ & $31(45 \%)$ & 0.026 & 2.5 \\
\hline Smoking habit(p/yr) & & & 0.001 & 8.2 \\
\hline No & $22(48 \%)$ & $59(86 \%)$ & & \\
\hline$<10$ & $6(13 \%)$ & $2(3 \%)$ & & \\
\hline $10-20$ & $1(2 \%)$ & $2(3 \%)$ & & \\
\hline$>20$ & $17(37 \%)$ & $6(8 \%)$ & & \\
\hline DLP & $36(78.2 \%)$ & $52(69.5 \%)$ & 0.065 & \\
\hline Statin usage & $16(35 \%)$ & $28(40.5 \%)$ & & \\
\hline HDL $<40$ & $9(19.5 \%)$ & $16(23.9 \%)$ & & \\
\hline LDL $>160$ & $5(11 \%)$ & $5(75 \%)$ & & \\
\hline TG $\geq 240$ & $6(13 \%)$ & $3(4.5 \%)$ & & \\
\hline $\mathrm{Hs}-\mathrm{CRP}(>700 \mathrm{ng} / \mathrm{ml})$ & $46(100 \%)$ & $69(100 \%)$ & 0.53 & \\
\hline
\end{tabular}


Table 2 Anthropometrics, comparison in case-control group, area under curve .

\begin{tabular}{|c|c|c|c|c|}
\hline & Cases $(\mathbf{n}=46)$ & Controls $(\mathbf{n = 6 9 )}$ & p-value & 0.38 \\
\hline Weight $(\mathrm{Kg})$ & $75.30( \pm 2.6)$ & $77.12( \pm 1.60)$ & 0.023 & 0.50 \\
\hline Height $(\mathrm{m})$ & $1.66( \pm 0.10)$ & $1.65( \pm 0.10)$ & 0.65 & 0.35 \\
\hline BMI $(\mathrm{Kg} / \mathrm{m} 2)$ & $27.33( \pm 6.30)$ & $28.33( \pm 5.20)$ & 0.21 & 0.42 \\
\hline WC & $100.00( \pm 15.30)$ & $103.01( \pm 10.50)$ & 0.001 & 0.33 \\
\hline HC & $93.90( \pm 17.50)$ & $104.2( \pm 10.50)$ & 0.67 & 0.46 \\
\hline TC & $46.10( \pm 6.10)$ & $46.7( \pm 6.70)$ & 0.00 & 0.70 \\
\hline WHR & $1.08( \pm 0.16)$ & $0.99( \pm 0.06)$ & 0.61 & 0.46 \\
\hline WTR & $2.19( \pm 0.24)$ & $2.20( \pm 0.25)$ & 0.19 & 0.38 \\
\hline
\end{tabular}

WC: Waist Circumference; HC: Hip Circumference; TC: Thigh Circumference; WHR: Waist to Hip Ratio; WTR: Waist to Thigh Ratio; WHtR: Waist to Height Ratio; AUC: Area Under Curve

Table 3 Different proposed cut-offs for waist-hip circumference ratio in T2DM patients (Male vs. Female).

\begin{tabular}{|c|c|c|c|}
\hline & Cut-off & Sensitivity & Specificity \\
\hline \multirow{2}{*}{ Male $(\mathbf{n = 8 4})$} & 0.962 & $79 \%$ & $20 \%$ \\
\hline \multirow{3}{*}{ Female(n=31) } & 1.000 & $69 \%$ & $60 \%$ \\
\hline & 1.05 & $42 \%$ & $84 \%$ \\
\hline & 1.00 & $92 \%$ & $82 \%$ \\
\hline & 1.02 & $90.9 \%$ & $84.2 \%$ \\
\hline
\end{tabular}

Table 4 Stepwise forward Regression for binary outcome, symptomatic PAD.

\begin{tabular}{|c|c|c|}
\hline & Odds Ratio & Confidence interval 95\% \\
\hline WHR $\geq 1$ & 7.71 & $2.86-20.7$ \\
\hline HTN & 4.75 & $1.69-13.32$ \\
\hline Smoking Habit & 2.48 & $1.58-3.88$ \\
\hline DM duration & & \\
\hline $5-10$ years & 6.25 & $1.2-31.2$ \\
\hline$>10$ years & 6.38 & $1.5-26.7$ \\
\hline
\end{tabular}

WHR: Waist-hip ratio; HTN: Hypertension; DM: Diabetes Mellitus

evaluated the previously suggested cut offs for WC in Iranian population by Esteghamati A. et al [18]. They suggested WC $>91.5$ in men and WC>85.5 in women as a criteria of metabolic syndrome. However in our study on patients with T2DM, it was not significantly related to symptomatic PAD ( $p$ value 0.62 and 0.45 , respectively). Waist-height ratio $\geq 0.5$ was inversely related to PAD (OR=0.095, Cl 95\% 0.02-0.45). Indeed, height more than twice the waist circumference was a strong predictor of PAD in T2DM patients (OR=10.52 Cl 95\% 2.21-50.15). TC and WTR were not significant (neither in men nor in women, $p$-value $>0.5$ ).

In multivariate binary logistic regression (Enter Method), after adjustment for Age, HTN and Smoking and duration of DM, WHR $\geq 1$ was still related to symptomatic PAD in patients with T2DM $(\mathrm{OR}=9.26, \mathrm{p}$-value $=0.000)$. Therefore, in our study major CVD risk factors did not confound the relation between WHR and PAD. All anthropometric indices in Table $\mathbf{2}$ were included in a binary logistic regression, when PAD was the outcome of the interst. WHR was still significantly related to symptomatic PAD in T2DM p-value=0.032, OR=5.46) Table 4.

All significant variables together, Stepwise forward regression was applied to achieve the best fitted regression model for
PAD prediction; at first the variable with the smallest $p$-value (waist-hip ration $\geq 1$ ) entered the model and so on. The age was removed from the model because its $p$-value became larger than the default limit ( $p$-value=0.1). In the final model, WHR $\geq 1, H T N$, duration of DM and Smoking were included. Patients with more than 10 years history of DM, had 6.38 fold greater risk of PAD than patients with $<5$ years of DM.

In present study, 32 patients had foot ulcers at the moment or suffered previously. Those with T2DM foot and $A B \mid>0.9$ were included in the control group $(n=10)$ and others were considered cases.

Data registry was not complete for DM treatment; oral vs. insulin. However, from total 86 patients, 46 patients ( 22 controls vs. 24 cases) were on Metformin and 40 patients ( 22 controls vs. 18 controls) were on Insulin therapy for their DM ( $p$ value=0.63).

\section{Discussion}

Disputations are still implied about the most accurate anthropometric index of obesity. On the other hand, the relation between these indices and other traditional CVD risk factors is challenging. In this study, we determined the pure effect of several anthropometric indices on the incident of symptomatic PAD, as a strong marker of CVD in T2DM patients.

Patients with type 2 diabetes mellitus may suffer from foot ulcers, with the life time prevalence as high as $25 \%$ [20]. In these patients, neuropathy and PAD are two of major risk factors of foot ulcer.

PAD is a progressive condition that leads to stenosis and occlusion of the arterial bed, and is a marker of systemic atherosclerosis. Both diabetes mellitus and PAD are big concerns in public health. The most prevalent risk factors for PAD are tobacco smoking and T2DM. Although much is known about PAD in general population, in T2DM patients the disease management and assessment is less clear [2]. In addition, many previous studies have reported PAD risk factors in general population, but fairly few have conducted their researches on T2DM patients with PAD.

The prevalence of PAD increases with age, mostly after age 40. Hence we included patients with T2DM (both cases and controls) older than 40 years old in our study.

In our study and previous similar articles [21] Smoking and hypertension were shown to have a great impression on PAD. Another 
important issue is an asymptomatic PAD which includes 20 to 50 percent of the patients [6]. In this study we evaluated symptomatic patients, and other asymptomatic patients were excluded.

Male type obesity (also known as abdominal obesity) increases the risk of CVD, T2DM and hypertension [22]. As mentioned above, after smoking, T2DM is the most important risk factor of PAD. Overweight and central obesity will increase the risk of T2DM, but the role of those, on PAD in T2DM is not clear yet. WC has been proposed to be a practical measure of intra-abdominal fat mass [23], but this measure does not take height into consideration and may lead to over- or underestimation of the risk for short or tall patients [17]. Recent studies have supported the $\mathrm{WHtR} \geq$ 0.5 as a good predictor for CVD risk factors [24], which in our study analysis it was inversely related to the outcome. Inverse relation between $\mathrm{BMI}$ and $\mathrm{WC}$ with cardiovascular event, after adjustment for other cardiovascular risk factors was reported [25]. In our study BMI was higher in controls but it was not statistically significant ( $p$-value $=0.051$ ).

BMI as a well-known indicator of general obesity has been debated since it does not predict the distribution of fat in the body. In our analysis, BMI was not associated with symptomatic PAD.

Obesity is more common among T2DM patients, so the cutoff value for every anthropometric index is different in this population. We did not aim to determine the appropriate cut-offs for each index because that kind of study needs larger sample size and also a cross sectional design. We tried our best to evaluate different anthropometric indices and their association with symptomatic PAD in T2DM patients.

In previous study in adult Nigerian patients with diabetes, none of the anthropometric indices (BMI, WHR and WC) were found significant [26]. The positive correlation between short stature and PAD was reported in Chinese type 2 diabetes patients [27] but was not significant in our study. In our study the cases were taller than controls ( $p$-value $>0.05$ ).

Female-type (gynecoid-type) obesity, when adipose tissue is predominantly distributed in the lower parts of the body (hip and thigh), has been shown to have less adverse impact on cardiovascular system $[28,29]$. Since it was debated that increase in hip circumference would diminish the risk for T2DM [30]. According to our data analysis, mean hip circumference was higher in controls (104.2 vs. 94.9 p-value=0.001), however its discriminative value was not as high as WHR.

High Sensitivity CRP was found to be relevant to PAD development and severity [31]. Diabetes per se, causes an inflammatory response in the body which may lead to atherosclerosis [32]. Elevated levels of hs-CRP is associated with higher risk of CVD and T2DM [33]. Such results are concordant with ours in which hsCRP was higher than normal range in all of the patients. The pure effect of hs-CRP on PAD was not investigated in present study.

Hyperlipidemia is another risk factor that favor the development of PAD [34], but in our study, it was not significantly different in cases and controls. It may results from the strong association between T2DM and dyslipidemia in both groups which concealed its pure effect on PAD.

Although history of cigarette smoking was significantly related to PAD, duration of smoking and also duration of T2DM were not significant variables in this equation. This result was also similar to the study in Pakestanian population [35].

We did not discuss different medical treatments in this study. Oral agents vs. Insulin therapy for diabetes, was not analyzed for all the patients because of many missing data. Statin therapy has an important part in PAD medical treatment, despite normal lipid profile. However, we did not take this concept into account in our analysis on lipid profile because we did not have the history of the patients' lipid profile before statin consumption.

As obese patients are more prone to T2DM, the previous cutoffs of obesity (and other proxies for general/abdominal obesity) may change in the future to assess the risk of CVD in this group. We think WHR is a simple measure of abdominal obesity and is related to PAD in diabetes mellitus.

All patients signed the informed consent before attending to the project. All the researchers were obligated to describe the benefits of this research for every individual volunteer.

\section{Acknowledgment}

Our gratitude goes to obesity clinic of Sina hospital and vascular surgery clinic in which this prospective case-control design was conducted. 


\section{References}

1 Vavra AK, Kibbe MR (2009) Women and peripheral arterial disease. Womens Health (Lond Engl) 5: 669-683.

2 (2014) Peripheral arterial disease in people with diabetes.

3 Fowkes FG (1988) The measurement of atherosclerotic peripheral arterial disease in epidemiological surveys. Int J Epidemiol 17: 248-254.

4 Premalatha G, Ravikumar R, Sanjay R, Deepa R, Mohan V (2002) Comparison of colour duplex ultrasound and ankle-brachial pressure index measurements in peripheral vascular disease in type 2 diabetic patients with foot infections. J Assoc Physicians India 50: 1240-1244.

5 Ashraf H, Rashidi A, Noshad S, Khalilzadeh O, Esteghamati A (2011) Epidemiology and risk factors of the cardiometabolic syndrome in the Middle East. Expert Rev Cardiovasc Ther 9: 309-320.

6 Hirsch AT (2006) 2005 Practice Guidelines for the management of patients with peripheral arterial disease (lower extremity, renal, mesenteric, and abdominal aortic): a collaborative report from the American Association for Vascular Surgery/Society for Vascular Surgery, Society for Cardiovascular Angiography and Interventions, Society for Vascular Medicine and Biology, Society of Interventional Radiology, and the ACC/AHA Task Force on Practice Guidelines (Writing Committee to Develop Guidelines for the Management of Patients With Peripheral Arterial Disease): endorsed by the American Association of Cardiovascular and Pulmonary Rehabilitation; National Heart, Lung, and Blood Institute; Society for Vascular Nursing; TransAtlantic Inter-Society Consensus; and Vascular Disease Foundation. Circulation 113: 463-654.

7 Althouse AD (2014) Risk factors for incident peripheral arterial disease in type 2 diabetes: results from the Bypass Angioplasty Revascularization Investigation in type 2 Diabetes (BARI 2D) Trial. Diabetes care 37: 1346-1352.

8 Guh DP, Zhang W, Bansback N, Amarsi Z, Birmingham CL, et al. (2009) The incidence of co-morbidities related to obesity and overweight: a systematic review and meta-analysis. BMC Public Health 9: 88.

9 Branca F, Nikogosian H, and Lobstein T (2007) The challenge of obesity in the WHO European Region and the strategies for response: summary. World Health Organization.

10 Power ML, Schulkin J (2008) Sex differences in fat storage, fat metabolism, and the health risks from obesity: possible evolutionary origins. Br J Nutr 99: 931-940.

11 Vasheghani-Farahani A (2013) Sagittal abdominal diameter to triceps skinfold thickness ratio: A novel anthropometric index to predict premature coronary atherosclerosis. Atherosclerosis 227: 329-333.

12 Canuto KJ (2011) Study protocol: a pragmatic randomised controlled trial of a 12-week physical activity and nutritional education program for overweight Aboriginal and Torres Strait Islander women. BMC public health 11: 655 .

13 Hajer GR, van Haeften TW, Visseren FL (2008) Adipose tissue dysfunction in obesity, diabetes, and vascular diseases. Eur Heart J 29: 2959-2971.

14 Dalton M, Cameron AJ, Zimmet PZ, Shaw JE, Jolley D, et al. (2003) Waist circumference, waist-hip ratio and body mass index and their correlation with cardiovascular disease risk factors in Australian adults. J Intern Med 254: 555-563.

15 Ross R, Berentzen T, Bradshaw AJ, Janssen I, Kahn HS, et al. (2008) Does the relationship between waist circumference, morbidity and mortality depend on measurement protocol for waist circumference? Obes Rev 9: 312-325.
16 (2000) Obesity: preventing and managing the global epidemic. Report of a WHO consultation. World Health Organ Tech Rep Ser 894: 1-253.

17 Browning LM, Hsieh SD, Ashwell M (2010) A systematic review of waist-to-height ratio as a screening tool for the prediction of cardiovascular disease and diabetes: $0 \cdot 5$ could be a suitable global boundary value. Nutrition research reviews 23: 247-269.

18 Esteghamati A, Ashraf H, Rashidi A, Meysamie A (2008) Waist circumference cut-off points for the diagnosis of metabolic syndrome in Iranian adults. Diabetes Res Clin Pract 82: 104-107.

19 Panel NC (2002) Third Report of the National Cholesterol Education Program (NCEP) Expert Panel on Detection, Evaluation, and Treatment of High Blood Cholesterol in Adults (Adult Treatment Panel III) final report. Circulation 106: 3143.

20 Boulton AJ (2008) Comprehensive foot examination and risk assessment a report of the task force of the foot care interest group of the American diabetes association, with endorsement by the American association of clinical endocrinologists. Diabetes care 31: 1679-1685.

21 Smith SC, Milani RV, Arnett DK, Crouse JR, McDermott MM, et al. (2004) Atherosclerotic Vascular Disease Conference: Writing Group II: risk factors. Circulation 109: 2613-2616.

22 Nguyen NT (2008) Association of hypertension, diabetes, dyslipidemia, and metabolic syndrome with obesity: findings from the National Health and Nutrition Examination Survey, 1999 to 2004. Journal of the American College of Surgeons 207: 928-934.

23 Pouliot MC (1994) Waist circumference and abdominal sagittal diameter: best simple anthropometric indexes of abdominal visceral adipose tissue accumulation and related cardiovascular risk in men and women. The American journal of cardiology 73: 460-468.

24 Lee CM, Huxley RR, Wildman RP, Woodward M (2008) Indices of abdominal obesity are better discriminators of cardiovascular risk factors than BMI: a meta-analysis. J Clin Epidemiol 61: 646-653.

25 Bechlioulis A, Vakalis K, Naka KK, Bourantas CV, Papamichael ND, et al. (2013) Paradoxical protective effect of central obesity in patients with suspected stable coronary artery disease. Obesity (Silver Spring) 21: E314-321.

26 Oyelade BO, Olaolorun AD, Odeigah LO, Amole IO, Aderibigbe SA (2014) The relationship between obesity and peripheral arterial disease in adult Nigerian diabetics. Niger Postgrad Med J 21: 57-60.

27 Fu X, Zhao S, Mao H, Wang Z, Zhou L (2015) Association of height with peripheral arterial disease in type 2 diabetes. J Endocrinol Invest 38: 57-63.

28 Janjic D (1996) [Android-type obesity and gynecoid-type obesity]. Praxis (Bern 1994) 85: 1578-1583.

29 Hartz AJ, Rupley DC, Rimm AA (1984) The association of girth measurements with disease in 32,856 women. Am J Epidemiol 119: 71-80.

30 Parker ED, Pereira MA, Stevens J, Folsom AR (2009) Association of hip circumference with incident diabetes and coronary heart disease: the Atherosclerosis Risk in Communities study. Am J Epidemiol 169: 837-847.

31 Maksimovic M (2010) Relationship between sociodemographic, anthropometric and biochemical characteristics and degree of peripheral arterial disease. Srpski arhiv za celokupno lekarstvo 138: 584-589. 
32 Schmidt MI, Duncan BB, Sharrett AR, Lindberg G, Savage PJ, et al. (1999) Markers of inflammation and prediction of diabetes mellitus in adults (Atherosclerosis Risk in Communities study): a cohort study. Lancet 353: 1649-1652.

33 Ridker PM (2006) A Symposium: the Interplay of Dyslipidemia and Inflammation: Reducing Cardiovascular Risk in Diverse Patient Populations: Based on a Satellite Symposium Held November 6,
2004, Prior to the American Heart Association Scientific Sessions 2004 in New Orleans, Louisiana. Elsevier.

34 Selvin E, Erlinger TP (2004) Prevalence of and risk factors for peripheral arterial disease in the United States: results from the National Health and Nutrition Examination Survey, 1999-2000. Circulation 110: 738-743.

35 Akram J, Aamir AU, Basit A, Qureshi MS, Mehmood T, et al. (2011) Prevalence of peripheral arterial disease in type 2 diabetics in Pakistan. J Pak Med Assoc 61: 644-648. 